\title{
US big-data health network launches aspirin study
}

\section{PCORI clinical-research initiative will collect information on some 30 million people.}

\section{BY SARA REARDON}

$\mathrm{O}$ ne of the largest big-data experiments in health care has set its first research target. The leaders of the Patient-Centered Outcomes Research Institute (PCORI) in Washington DC voted on 29 July to focus the institute's first clinical trial on the use of aspirin to prevent heart disease. The US $\$ 10$-million pilot study will be conducted through PCORnet, a network set up by PCORI to collect health-care data such as insurance claims, blood tests and medical histories for as many as 30 million people in the United States.

PCORnet is the latest - and largest - of a number of initiatives attempting to use patient data to improve health care. Private companies such as Kaiser Permanente in Oakland, California, and medical networks such as Partners HealthCare in Boston, Massachusetts, have similar aims. PCORI was established in 2010 with more than $\$ 3$ billion from the US government to fund research comparing the effectiveness of treatments, and has so far given out $\$ 549$ million in grants (see 'Network profile'). Its own project, PCORnet, comprises 29 smaller networks of US hospitals and patient groups. The aspirin trial is part of a pilot phase to work out questions such as how best to recruit study volunteers, standardize their records and build rapport with them.

In the second phase, scheduled to begin in September 2015, outside scientists will be able to mine PCORnet data for their research. "My measure of success will be the number of questions we get asked," says Christopher Forrest, a paediatrician at Children's Hospital of Philadelphia in Pennsylvania, who leads one of the networks participating in PCORnet.

The aspirin trial is scheduled to launch in early 2015. Participants will take daily doses of aspirin that fall within the range typically prescribed for heart disease, and be monitored to determine whether one dosage works better than the others. "While this study is a proof of concept, [aspirin dosage] is a very important question as well," says PCORI director Joe Selby. "We're proving that you can ask and answer questions that matter to patients, and this one has gone many years without an answer.'

PCORI does not aim to test new potential treatments or uncover disease mechanisms that falls within the purview of agencies such

\section{DATA POINTS}

Network profile

The Patient-Centered Outcomes Research Institute (PCORI) is setting up a database of US health records that will be used to compare the effectiveness of different medical treatments. The system, PCORnet, will connect multiple smaller networks, giving researchers access to records at a large number of institutions without creating a central data repository. Here are some key facts about PCORnet and its parent institution:

- Initial funding for PCORnet: US\$93.5 million

- Number of member networks: 29

- Total number of patient records:

up to $\mathbf{3 0}$ million

- Budget for pilot study of aspirin dosage: $\$ 10$ million

- Number of projects funded by PCORI

since 2012: 313

- Amount of funding awarded by PCORI since 2012: $\$ 549$ million

as the US National Institutes of Health. "I see them as complementing, rather than replacing, randomized clinical trials," says William Hersh, a biomedical informatician at Oregon Health \& Science University in Portland who is not involved in PCORI. One of the greatest challenges will be standardizing data from different networks to enable accurate comparison, he says. The many types of data - scans from medical imaging, vital-signs records and, eventually, genetic information - can be messy, and record-keeping systems vary between health-care institutions.

\section{ADVICE AND CONSENT}

Transparency in how patients' records are used will be essential for the programme to succeed, says Sam Smith, an activist at medConfidential, a UK-based organization that campaigns for patient privacy protection. "Generally if you tell people where their data are going, they're a lot happier than if you don't," he says. Transparency issues have plagued the UK National
Health Service's care.data, a medical-records database whose launch has been delayed amid concerns about data security and informed consent.

To avoid similar pitfalls, PCORI says that patient representatives will help to review its grant applications and work with investigators on trials. But simplifying scientific concepts for non-specialists can be hard, says Glenn Cohen, a bioethicist at Harvard University in Cambridge, Massachusetts. "It has a certain public spirit, but expecting patients to be involved raises challenges when these policy questions have a technical underlying element," he says. Selby says that PCORI plans to work out ways to deal with such issues over the next year.

To ensure privacy, PCORnet will not collect personal data. Patients' records will be kept by their health-care providers. When outside researchers use PCORnet for studies, the relevant data can be analysed within the network and the results sent to the researcher, or anonymized and provided in raw form.

The aspirin study will use standard procedures for obtaining informed consent, in which participants are told specifically about the research question their data will be used to address. But the process will be more complicated when outside researchers use PCORnet's data. People may tire quickly of signing forms every time a researcher wants to use their records for a study. "If very explicit consent is required, cost and practicality are probably going to make it a non-starter," Cohen says. The more transparency, patient involvement and data security that PCORI can provide, he says, the more ethically sound it would be to forgo standard informed-consent procedures.

\section{CORRECTIONS}

In the News story 'Biosafety controls come under fire' (Nature 511, 515-516; 2014) the 'European Biosafety Organization' should have been the 'European Biosafety Association'. And the story 'Project drills deep into coming quake' (Nature $\mathbf{5 1 1}$, $516-517$; 2014) gave the old name for the Institute of Geological and Nuclear Sciences — it is now GNS Science. 